\title{
Efecto de la implementación de una "Red de Apoyo Social Adventista" en la habilidad del cuidado de los familiares cuidadores de personas con enfermedad crónica del Centro Poblado Virgen del Carmen - La Era, 2010.
}

\author{
Roussel Dávila Villavicencio
}

\begin{abstract}
RESUMEN
Objetivo: Determinar el efecto de la implementación de una "Red de Apoyo Social Adventista" en la habilidad del cuidado de los familiares cuidadores de personas con enfermedad crónica del Centro Poblado Virgen del Carmen La Era. Metodología: El estudio fue pre-experimental con pre test y post test, para lo cual se empleó el inventario de habilidad de Cuidado de Nogozi Nkongo. Participaron veinte familiares cuidadores que recibieron el acompañamiento social de veinte voluntarios y participaron en un programa educativo desarrollado en nueve semanas. Resultados: Los resultados obtenidos fueron positivos considerando que antes de la intervención el puntaje promedio de la habilidad de cuidado fue de 91.80 (nivel mediano) y después de la intervención el puntaje promedio fue de 118.45 (nivel alto), con una significancia de diferencia de medias de 0.000. Conclusiones: Se concluye que la implementación de una Red de Apoyo Social Adventista mejoró significativamente la habilidad del cuidado de los familiares cuidadores.
\end{abstract}

Palabras clave: Habilidad del cuidado, enfermedad crónica, familiares, apoyo social.

\begin{abstract}
Objective: to determine the implementation effect of the "Adventist Social Support Network" in the caring skill by family caregivers for people with chronic illnesses who belong to the "Virgen del Carmen-La Era" community. Methods: The study was pre-experimental performing the pretest and the posttest, using the Nkongo Nogozi Care Skills Inventory.Twenty family caregivers participated in this study who received social support from another twenty volunteers who offered them an educational program that lasted for nine weeks. Results: The results were positive whereas before the intervention the average score of caring skills were of 91.80 (medium level) and after the intervention the average score was 118.45 (high level), with as significance of mean difference of 0.000 . Conclusions: It is concluded that the implementation of an Adventist Social Support Network significantly improved the skill for family caregivers.
\end{abstract}

Keywords: Ability of care, chronic disease, family, social support

${ }^{1}$ Licenciado en Enfermería, Universidad Peruana Unión. 


\section{INTRODUCCIÓN}

El perfil epidemiológico de América Latina indica que en muchos países las enfermedades crónicas comparten los primeros lugares de morbimortalidad con tendencia a incrementarse. Este hecho, junto a la evasión de responsabilidades de cuidado formal para las personas en situación de enfermedad crónica, pone de manifiesto el problema del cuidado de largo término (Sánchez, 2002).

La Organización Mundial de la Salud (2008), manifiesta que el $60 \%$ de todas las defunciones, se deben a enfermedades crónicas como cáncer, diabetes, enfermedades neurológicas, hipertensión y enfermedades cardiacas, de este porcentaje los países con ingresos bajos y medios aportan el $80 \%$ de las muertes.

Velázquez (2009), en el estudio nacional de carga de enfermedad y lesiones, indica que el Perú ha presentado una transición epidemiológica, porque se ha demostrado que la mayor carga de enfermedad en el Perú se le atribuye a las enfermedades no transmisibles (58.5\%) y en segundo lugar a las enfermedades transmisibles maternas y perinatales $(26.8 \%)$, en tanto que los accidentes y lesiones aportan el $14.7 \%$ a la carga de enfermedad. En este sentido queda evidenciado que las enfermedades no transmisibles generan una mayor utilización de los servicios de salud, encarecen la atención y exigen mayor capacidad resolutiva de los establecimientos.

A su vez, estas enfermedades ocasionan serios efectos en la calidad de vida de quienes la padecen, sus familiares y la comunidad en general, sin distinción de sexo, raza o religión. El impacto que estas ocasionan dependen del tipo y estado de la enfermedad (inicial o terminal), estructura familiar (familia completa, madre soltera, hijo único), rol de la persona enferma crónica (hijo, abuelo, padre), ciclo vital individual y familia (criando niños, ancianos).

Es importante resaltar que la enfermedad crónica produce una dependencia paulatina e irreversible de tipo orgánico y social por lo que nunca se retorna a la categoría de sujeto sano. La persona pierde lentamente sus desempeños fisiológicos hasta que es incapaz de realizarlos por sí mismo; de igual manera, su dimensión social sufre pérdidas que se objetivan, sobre todo, en la ejecución deficiente de sus roles de acuerdo a los estándares sociales y en la incapacidad de ser recíproco en el sistema de intercambios sociales (Kittay, 1999). Esta condición lo obliga a confiar en el cuidado provisto por otros.

El cuidador es un familiar que toma decisiones y asume responsabilidades del cuidado con respecto a un ser querido que vive en situación de enfermedad crónica. Tomar decisiones es una actividad de gran trascendencia, sobre todo en este caso en que el cuidador debe decidir no solo sobre acciones de la vida diaria, sino incluso sobre el pronóstico de la enfermedad. Los cuidadores surgen de la necesidad de ayudar a un ser querido, un hijo, un padre, una madre, que padecen una enfermedad que necesita de cuidados permanentes; otros cuidadores responden espontáneamente a esta necesidad debido a que sienten un amor incondicional por esa persona; otros se vuelven cuidadores porque en la familia no hay otra persona que asuma ese rol (Sánchez, Pinto, Carrillo \& Barrera 2008).

Estover (1998), en su estudio titulado "Efectividad de una intervención de consejería para ayudar a los cuidadores familiares de pacientes crónicamente enfermos", señala que en las dos últimas décadas se ha observado un crecimiento en el número de familias con la responsabilidad de cuidar a pacientes con enfermedades crónicas; exponiéndose a varios riesgos ya que las tareas del familiar cuidador se vuelven cada vez más complejas y les demanda mayor cantidad de tiempo afectando su salud física y mental, llegando en muchos casos a desistir de dicha responsabilidad. Asimismo, la carga de estrés se intensifica cuando el cuidador no tiene la preparación adecuada, ni las alternativas de cuidado en el hogar tal como lo afirma Barrera (2005).

En este sentido, la necesidad de trabajar con los cuidadores de pacientes con enfermedad crónica se centra en el desarrollo de la habilidad de cuidado en estas personas, para generar en el paciente condiciones propicias de un mejor bienestar y una mejor calidad de vida. Al respecto, Nkongho (1999), menciona que el cuidado es un potencial presente en todas las personas, el cual es multidimensional y se puede aprender y cuantificar. La cuantificación de la habilidad de cuidado debe incluir aspectos cognitivos y actitudinales y se pueden medir según indicadores de conocimiento, valor y paciencia.

Las Redes de Apoyo Social son prácticas simbólicas culturales que incluyen el conjunto de relaciones interpersonales que integran a una persona con su entorno social y le permite mantener o mejorar su bienestar material, físico y emocional evitando así el deterioro real o imaginado que podría generarse cuando enfrenta dificultades, crisis o conflictos (Saranson 1983). Estas redes de apoyo permiten la existencia o disponibilidad de personas con las cuales se puede contar en períodos de necesidad; quienes proveen al individuo de amor y valor personal.

Cuando se habla de redes sociales, está implícita la idea de intercambio de apoyos, que constituye la esencia del funcionamiento de las redes. No obstante, "hay que tener presente que la importancia de las redes de 
relaciones varía en el tiempo y en el espacio; en coyunturas específicas pueden ser muy importantes, pero en otras son menos relevantes" (Oliveira \& Salles, 1989).

Una estrategia que puede ayudar a mejorar las habilidades de cuidado de los cuidadores familiares, es la capacitación que éstos reciben a través de los prestadores de salud o mediante los programas articulados al sistema formal de servicios de salud. Sin embargo, existe poca experiencia respecto a la implementación de redes de apoyo que surjan como iniciativa de la comunidad organizada y/o representaciones civiles tales como municipios, clubes, iglesias, entre otros. Las redes de apoyo comunitarias pueden brindar soluciones a demandas sociales específicas y se focalizan en la experiencia colectiva; pueden surgir alrededor de una institución, un centro de salud, una iglesia o una escuela. Esto, hace que las personas se conviertan en protagonistas de su propia vida, con capacidad de transformación histórica y ubicados en su contexto geográfico (CELADE, 2003).

Diversos estudios han mostrado cómo la integración social y las relaciones sociales pueden lograr un efecto positivo sobre la salud (Puga, et al., 2007). La red social puede ser un factor de protección frente a la pérdida de funcionalidad y el inicio de la discapacidad básica del paciente y asimismo de la propia familia que deberá afrontar esta nueva situación.

Estos beneficios grupales responden a dos claves de éxito, la primera según Tajfel (1984), se refiere a la identificación social que ésta produce en los miembros del grupo considerando que permite satisfacer necesidades comunes mediante la búsqueda de estrategias para resolver conflictos, mejorar el control y dominio de la realidad, así como para obtener consejo (Díaz \& Ferri, 2002). La segunda clave de éxito radica en la aceptación y comprensión entre sus miembros, así como el intercambio de información sociocognitiva por ejemplo, cuando en sus conversaciones transmiten conocimientos personales al grupo (Solomon, Pistrang \& Barker, 2001).

En consecuencia, la formación de redes de apoyo social que surjan como iniciativa en una comunidad, constituyen una estrategia poderosa para mejorar las habilidades de cuidado de los familiares con pacientes crónicos, generando un espacio para compartir información, experiencias de cuidado y manejo de situaciones difíciles dentro del hogar.

El Centro Poblado de Virgen del Carmen - La Era tiene una población adulta de 4,955. Según datos obtenidos de la Oficina de Epiedmiología de la Red 8 de Lima Este, durante el año 2007 se atendieron en este Centro de Salud 27 casos de enfermedades crónicas como cáncer, diabetes, hipertensión y enfermedades cardiovasculares; durante los meses de enero a setiembre del 2009, los casos nuevos se incrementaron a 40. Cabe indicar que actualmente en el Centro Poblado en mención, no existe un programa educativo que permita mejorar la habilidad de cuidado de los familiares de personas con enfermedad crónica, tampoco existe una red de apoyo social como iniciativa de los prestadores de servicios de salud y mucho menos por parte de la comunidad organizada como asociaciones, municipio e iglesias.

La Iglesia Adventista del Séptimo Día (IASD) es una organización religiosa, que tiene como uno de sus principios fundamentales el servicio al prójimo y cuenta en dicha comunidad con 1000 miembros, que en su mayoría $(70 \%)$ son jóvenes universitarios que voluntariamente desarrollan una serie de actividades religiosas y de proyección social (Secretaría de Iglesia Adventista Unión Norte, 2010). Después de haber realizado entrevistas con algunos líderes de los jóvenes adventistas de dicha localidad, éstos han expresado su deseo de participar voluntariamente en programas de salud orientados a la comunidad de manera organizada como parte de las actividades misioneras.

Ante el incremento progresivo de enfermedades crónicas en la localidad de Virgen del Carmen La Era, la ausencia de un programa orientado a desarrollar las habilidades de cuidado de los familiares con enfermedad crónica y el potencial de los miembros voluntarios de la Iglesia Adventista de dicha comunidad, surge la necesidad de poner a prueba una novedosa estrategia sanitaria local, que consiste en la implementación de una red de apoyo social teniendo como base organizativa a miembros de la Iglesia Adventista del Séptimo Día. En este sentido, se planteó como objetivo de investigación: Determinar el efecto de la implementación de una "Red de Apoyo Social Adventista" en la habilidad de cuidado de los familiares cuidadores de personas con enfermedad crónica del Centro Poblado Virgen del Carmen - La Era, durante el año 2010.

\section{MATERIAL Y MÉTODOS}

El estudio fue de tipo cuantitativo, de diseño pre experimental con pre prueba y pos prueba. Es importante mencionar que antes de la implementación de la Red de Apoyo Social Adventista, se realizó la medición de las habilidades de cuidados en los familiares cuidadores de personas con enfermedad crónica que forman parte del grupo de estudio. Luego de la implementación de dicha red con sus componentes respectivos, se realizó una segunda medición de las habilidades de cuidados en los familiares cuidadores, para su comparación con el test de inicio. De esta forma se pudo verificar si la variación del conocimiento, valor, paciencia 
y la habilidad en general fueron significativas respecto al pretest.

La población estuvo constituida por 20 familiares cuidadores de personas con enfermedad crónica que reciben atención ambulatoria en el Centro de Salud de la Era y cumplieron con los siguientes criterios: familiares cuidadores permanentes de pacientes que fueron diagnosticados con enfermedad crónica con presencia de discapacidad desde hace seis meses o más y que además aceptaron voluntariamente participar en el estudio evidenciado en la firma del documento de consentimiento informado.

La recolección de datos se realizó en dos momentos, durante la primera y novena semana, es decir antes y después de la intervención con la implementación de la Red de Apoyo Social Adventista y en ambos casos se realizó mediante la aplicación del Inventario de Habilidades de Cuidados (CAI) de Ngozy O. Nkongh, que consta de 37 items y de traducción oficial de inglés al español. Este instrumento tiene una validez general de 0.84 y una confiabilidad general de 0.80 . Los valores de las respuestas del inventario son de 1 a 4, donde 1 equivale a nunca, 2 casi nunca, 3 casi siempre y 4 siempre. Midiendo así las habilidades de cuidados en las categorías o dimensiones de conocimiento, valor y paciencia.

Con el propósito de realizar un análisis comprensible y significativo de la información recolectada a través de la aplicación del instrumento (Inventario de Habilidades de los Cuidados), éstos fueron trasladados a una plataforma matriz del software SPSS, el cual es un sistema amplio y flexible de análisis estadístico y de gestión de datos en un entorno gráfico.

Tabla 1

Nivel de conocimiento que tienen los cuidadores de sí mismos y de la persona que cuidan antes y después de participar en La Red de Apoyo Social Adventista en el Centro Poblado Virgen del Carmen - La Era, 2010.

\begin{tabular}{lcccc}
\multicolumn{1}{c}{ Nivel de conocimiento } & N & Antes & $\mathrm{N}^{\mathrm{o}}$ & Después \\
\hline Bajo & 2 & $10 \%$ & - & - \\
Mediano & 18 & $90 \%$ & 3 & $15 \%$ \\
Alto & - & - & 17 & $85 \%$ \\
Media de puntuación en el CAI & 36.00 (Mediano) & 48.40 (Alto) & \\
\hline Nivel Sig. Diferencia medias (95\%) & \multicolumn{3}{c}{0.000} \\
\hline
\end{tabular}

Antes de la implementación de la Red de Apoyo Social Adventista se encontró que el nivel de conocimiento que tienen los cuidadores de sí mismos y de las personas a quien dedican su cudado fue bajo en el 10\% de los cuidadores identificados y 90\% demostraron un nivel de conocimiento mediano. Sin embargo, después de la implementación de la Red de Apoyo y de la participación de los cuidadores en esta Red, se evidenció que 85\% de los cuidadores presentó un nivel alto y solo $15 \%$ presentó un nivel mediano. Asimismo, la tabla 1, presenta que el promedio de puntuación obtenido para la escala de conocimiento mediante la aplicación del CAI, en donde se observa que antes de la implementación de la Red de Apoyo Social Adventista, la media para esta escala fue de 36.00, ubicándose en un nivel mediano. En contraste con la media después de la implementación de dicha Red de Apoyo que se incrementó a 48.40, ubicándose en un nivel alto, mostrando una significancia de 0.000 en la prueba de diferencia de medias.

Tabla 2

Nivel de valor de los cuidadores para enfrentar el cuidado de su familiar antes y después de participar en La Red de Apoyo Social Adventista en el Centro Poblado Virgen del Carmen - La Era, 2010.

\begin{tabular}{lcccc}
\multicolumn{2}{c}{ Nivel de valor } & \multicolumn{2}{c}{ Antes } & \multicolumn{2}{c}{ Después } \\
\hline Bajo & $\mathrm{N}^{\text {o }}$ & $\%$ & $\mathrm{~N}^{\text {0 }}$ & - \\
Mediano & 11 & $55 \%$ & 12 & $60 \%$ \\
Alto & 09 & $45 \%$ & 08 & $40 \%$ \\
Media de puntuación en el CAI & 27.65 (Mediano) & 35.25 (Mediano) & \\
\hline Nivel Sig. Diferencia medias (95\%) & \multicolumn{3}{c}{0.000} \\
\hline
\end{tabular}


La tabla 2 muestra que respecto a valor de los cuidadores para afrontar la experiencia del cuidado, antes de la implementación de la Red de Apoyo Social Adventista, el 55\% presentó un nivel bajo, 45\% un nivel mediano y ningún cuidador presentó un nivel alto. Los datos que se muestran luego de la intervención con la Red de Apoyo, indican que un porcentaje significativo de los cuidadores (40\%) presentó un nivel de valor alto y el porcentaje de cuidadores con nivel de valor mediano se incrementó a $40 \%$, no presentándose cuidadores con nivel de valor malo. Cabe indicar que el promedio de las puntuaciones en la escala de valor antes de la implementación de la Red de Apoyo fue de 27.65 y después de dicha implementación se incrementó a 35.25, con una significancia de la prueba de diferencia de medias de 0.000 .

\section{Tabla 3}

Nivel de Paciencia de los cuidadores antes y después de participar en La Red de Apoyo Social Adventista en el Centro Poblado Virgen del Carmen- La Era, 2010.

\begin{tabular}{lcccc} 
Nivel de paciencia & \multicolumn{2}{c}{ Antes } & \multicolumn{2}{c}{ Después } \\
& $\mathrm{N}^{\circ}$ & $\%$ & $\mathrm{~N}^{\circ}$ & - \\
\hline Bajo & - & - & 02 & $10 \%$ \\
Mediano & 16 & $80 \%$ & 18 & $90 \%$ \\
Alto & 04 & $20 \%$ & \\
Media de puntuación en el CAI & 28.15 (Mediano) & 34.80 (Alto) & \\
\hline Nivel Sig. Diferencia medias (95\%) & \multicolumn{2}{c}{0.000} \\
\hline
\end{tabular}

En cuanto al nivel de paciencia, la tabla 3 revela que antes de la intervención solo $20 \%$ presentó un nivel alto y después de la intervención este porcentaje se incrementó a 90\%. Por otro lado, $80 \%$ de los cuidadores presentaron un nivel de paciencia mediano antes de la intervención y luego este porcentaje se redujo a solo $10 \%$. El promedio de puntuaciones en la escala paciencia antes de la implementación de la Red de Apoyo Social Adventista fue de 28.15, ubicándose en un nivel mediano, mientras que después de la implementación de la Red de Apoyo, el promedio fue de 34.80, ubicándose en un nivel alto. La prueba de diferencia de medias tuvo un valor de significancia de 0.000 .

Tabla 4

Nivel de habilidad de cuidado de los familiares cuidadores de personas con enfermedad crónica antes y después de su participación en la Red de Apoyo Social Adventista del Centro Poblado Virgen del Carmen - La Era, 2010

\begin{tabular}{lcccc}
\hline \multirow{2}{*}{ Nivel de habilidad de cuidado } & \multicolumn{2}{c}{ Antes } & \multicolumn{2}{c}{ Después } \\
& $\mathrm{N}^{\mathrm{o}}$ & $\%$ & $\mathrm{~N}^{\mathrm{o}}$ & - \\
\hline Bajo & 01 & $05 \%$ & 04 & $20 \%$ \\
Mediano & 19 & $95 \%$ & 16 & $80 \%$ \\
Alto & - & - & 118.45 (Alto) \\
Media de puntuación en el CAI & 91.80 (Mediano) & \multicolumn{3}{c}{0.000} \\
\hline Nivel Sig. Diferencia medias (95\%) & & \\
\hline
\end{tabular}

La Tabla 4 presenta los resultados generales respecto a la habilidad de cuidado de los familiares cuidadores de personas con enfermedad crónica, donde se observa que antes de la intervención con la implementación de la Red de Apoyo Social Adventista no se encontró a ningún cuidador con nivel alto de habilidad de cuidado, mientras que después de la implementación de la referida Red, $80 \%$ de los cuidadores presentaron un nivel alto de habilidad de cuidado. Asimismo, antes de la implementación 95\% presentó un nivel mediano de habilidad de cuidado siendo que después de la implementación este porcentaje se redujo a 20\%, llegando finalmente a no presentar cuidadores con nivel bajo de habilidad para el cuidado. Esta misma tabla muestra que el promedio general obtenido mediante la aplicación del CAI fue de 91.80, que ubica a la habilidad de cuidado de los cuidadores en un nivel mediano, contrario a esto luego de la implementación de la Red de Apoyo, el promedio general se incrementó a 118.45, ubicando a la habilidad de los cuidadores en un nivel alto, con una significancia de la prueba de diferencia de medias de 0.000. Es importante indicar que durante el proceso de capacitación que tuvo una duración de 9 semanas se contó con 
el $100 \%$ de asistencia y participación de voluntarios y cuidadores, quienes al final de los talleres manifestaron su agradecimiento por los beneficios obtenidos y que estarían gustosos de participar activamente programas similares, observando además que ningún voluntario y cuidador se retiró del programa.

\section{DISCUSIÓN}

Como parte de los resultados generales destaca el hecho que el $90 \%$ de los cuidadores son de sexo femenino, lo cual coincide con hallazgos evidenciados por investigadores como Vega (2006) y Parra (2006), quienes encontraron en sus estudios, que del total de cuidadores, más del $80 \%$ son mujeres y de este modo destacan el meritorio papel protagónico del sexo femenino en el rol del cuidado. Respecto a esto, Bazo (1998), manifiesta que el cuidado familiar se resuelve fundamentalmente a costa del trabajo y el tiempo de las mujeres, es decir, el cuidado familiar "se escribe en femenino singular" (Rodriguez, 1998).

En este sentido, las mujeres siguen siendo las principales cuidadoras de sus familiares dependientes en el domicilio. La prestación de cuidados no se reparte, habitualmente, de forma equitativa entre los miembros de la familia. Existe un familiar denominado "cuidador familiar" o "cuidador informal", que es aquel sobre el que recae la mayor responsabilidad del cuidado. Este cuidador informal ha sido definido como la persona que proporciona la mayor parte de la asistencia y apoyo diario a quien padece una enfermedad y también la que permite seguir viviendo en su entorno y de una manera confortable y segura a una persona que por razones de edad o incapacidad no es totalmente independiente (Philip y Young, 1988; De la Rica, Hernando, 1994).

Los autores anteriormente mencionados también indican que estas tareas de cuidados recaen fundamentalmente en las mujeres de las generaciones intermedias, y entran en contradicción directa con las demandas derivadas del cambio que se está produciendo en el rol social de las mujeres, que se están incorporando de manera activa al mundo del trabajo retribuido. En este sentido, cabe destacar que el presente estudio revela que un importante grupo de cuidadoras $(40 \%)$ se encuentran entre las edades de 25 a 31 años, que tal como se ha referido se encuentra en una etapa denominada de contradicción en su rol social y productivo lo que podría repercutir en su realización como personas.

En cuanto a los resultados específicos del presente estudio se encontró que antes de la implementación de la Red de Apoyo Social Adventista la mayoría (90\%) de los cuidadores presentaron un nivel mediano de conocimiento de sí mismos y de la persona con enfermedad crónica a quien cuida con un promedio de puntuación en dicha escala de 36.00. Después de la intervención, el conocimiento de la mayoría de los cuidadores fue Alto y el promedio de puntuaciones en la escala se incrementó en 48.40, siendo la significancia de la prueba estadística de diferencia de medias antes-después de 0.000 , lo cual permite afirmar que el nivel de conocimiento mejoró después de la intervención con la implementación de la Red de Apoyo Social Adventista (tabla 1).

Referido al conocimiento como parte de la habilidad de cuidado se debe tener en cuenta que éste es considerado como una condición primordial para cuidar, éste incluye el autoconocimiento, el conocimiento del otro, sus fortalezas y limitaciones al igual que sus necesidades particulares; y el ritmo alterno está relacionado con las fluctuaciones o cambios que se dan durante la experiencia del cuidado. El conocimiento implica el entendimiento con la persona de sus necesidades, fuerzas, debilidades y lo que refuerza su bienestar, puede ser implícito o explícito, directo e indirecto, conociendo sus fuerzas y limitantes (Ngozi, 1999).

Otra dimensión fundamental en la habilidad de cuidado, lo constituye el valor para afrontar el cuidado del familiar que presenta enfermedad crónica. El valor se entiende como la fuerza para apoyar a otro, se trata de la fuerza interna que permite a las personas dar el cuidado fortaleciéndose y creciendo como cuidadores (Koffman, 1997). Tomando como referencia lo anteriormente planteado, se debe indicar que en el presente estudio se ha podido obtener como resultado (tabla 2) que antes de la implementación de la Red de Apoyo Social Adventista el valor para enfrentar la tarea del cuidado al familiar fue de nivel bajo en la mayoría de los cuidadores (55\%) y $45 \%$ presentó un nivel mediano en esta dimensión, no existiendo cuidadores con nivel de valor alto. Luego de la implementación no se presentó ningún cuidador con nivel de valor bajo, $60 \%$ presentó un nivel mediano y $40 \%$ obtuvo un nivel alto. Asimismo, el promedio de puntuación se incrementó de 27.65 (mediano) a 35.25 (mediano), que si bien como promedio no logró llegar a un nivel alto la prueba estadística de diferencia medias antes-después muestra una significancia de 0.000 , lo que permite afirmar que existió un incremento del valor en los cuidadores para enfrentar la loable tarea de cuidar a un familiar con enfermedad crónica gracias a la implementación de la Red de Apoyo Social Adventista, mediante el cual los participantes tuvieron un espacio para reflexionar sobre sus dudas, 
su estrés y sus responsabilidades frente a la situación de cuidado, eliminando o controlando los sentimientos de culpa y la impotencia que ejercen un fuerte impacto en la disminución de la fuerza interna que requiere el cuidador (Koffman, 1997).

La tercera dimensión de la habilidad de cuidado lo constituye la paciencia. Según Ngozi (1999), la paciencia es otro componente en la adquisición de la habilidad para el cuidado, afirma además, que la paciencia es dar tiempo y espacio para la autoexpresión y exploración, incluye el grado de tolerancia ante la desorganización y problemas que limitan el crecimiento de sí mismos y de otros.

Los resultados del presente estudio (tabla 3), revelan que antes de la implementación de la Red de Apoyo Social Adventista la mayoría de cuidadores $(80 \%)$ presentó un nivel mediano y solo $20 \%$ presentó un nivel Alto. En contrate se muestra que luego de la implementación de la Red de Apoyo Adventista la mayoría de los cuidadores (90\%) presentaron un nivel Alto de paciencia y $10 \%$ presentaron un nivel mediano. La prueba de diferencia de medias arroja un promedio de puntuación en la escala de paciencia antes de la implementación de la Red de Apoyo de 28.15 (mediano), siendo que después de la implementación de la referida Red, el promedio se incrementó a 34.80 (Alto). Considerando que el valor de la significancia de la prueba de diferencia de medias es de 0.000 , por lo cual afirmarmos que sí se produjo un incremento significativo de la paciencia en los cuidadores.

Los resultados generales de la habilidad de cuidado de los cuidadores de personas con enfermedad crónica antes de la implementación de la Red de Apoyo Social Adventista (tabla 4), muestran que un importante porcentaje de cuidadores presentaban un nivel mediano de habilidad (95\%), cifra que sufrió una variación importante considerando que después de la intervención con la Red de Apoyo $80 \%$ de los cuidadores presentaron un nivel alto de habilidad y solo $20 \%$ un nivel mediano. Además la media de puntuaciones que arrojó el CAI antes de la intervención (91.80) tuvo un importante incremento después de la intervención (118.45). Esta variación fue estadísticamente significativa (sig. 0.000), por lo que se afirma que la habilidad de cuidado de los cuidadores mejoró gracias a la implementación de la Red de Apoyo Social Adventista. De este modo, la mayoría de los cuidadores alcanzaron el nivel de desarrollo y crecimiento que les permitió vivir la experiencia de cuidado con autenticidad. Es necesario remarcar entonces que los niveles óptimos de habilidad de cuidado lleva a los cuidadores a experimentar una sensación de pertenencia, vínculo y estabilidad que les permite mayor resistencia al estrés (Ngozi, 1999).
Los resultados evidenciados en el presente estudio han sido mejores a algunas experiencias previas que solo incluían el componente educativo o de capacitación, tal es así que Vega, Mendoza, Ureña y Villamil (2008) en su estudio titulado efectividad de un programa educativo en la habilidad de cuidado de familiares cuidadores de personas en situación crónica de enfermedad, el mismo que fue realizado en una muestra de 40 personas que ejercían el rol de cuidadores de pacientes que se atendían en el Centro de Rehabilitación Cardioneuromuscular de San José de Cúcuta, presentaron como conclusión que el programa permitió mejorar la habilidad general y la categoría valor, sin embargo las categorías de conocimiento y paciencia presentaron un ligero incremento, no alcanzando estadísticamente el resultado esperado.

La estrategia que ha permitido realizar la intervención en el Centro Poblado Virgen del Carme, La Era, con los resultados anteriormente comentados ha sido la Implementación de una Red de Apoyo Social Adventista. En este sentido, se debe mencionar que dicha estrategia se sustenta en la concepción de que las organizaciones comunales y sociales son determinantes como medio de contención y pilares de apoyo; en la realidad éstas procuran alternativas de ayuda, lo cual sugiere que el mayor trabajo debería estar dirigido a la orientación y el reforzamiento de estos grupos para que puedan resolver localmente las situaciones que se presentan.

Por los resultados obtenidos, se corrobora lo que afirma Khan y Antomucci, (1980), citados por CELADE (2003), al referir que los apoyos sociales son las transacciones interpersonales que implican afecto, ayuda y afirmación; constituyen un flujo de intercambio de recursos, acciones e información. Ante el declive funcional, cumplen un papel protector, previenen la enfermedad y mantienen un estado adecuado de salud y de bienestar personal. En síntesis, contribuyen a superar mejor las crisis que se presentan.

Bajo el enfoque anteriormente mencionado la implementación de la Red de Apoyo conformado por miembros de la Iglesia Adventista del Séptimo Día (IASD) comprometidos con la misión de proclamar el evangelio mediante el servicio, ha constituido una experiencia novedosa para beneficio de los familiares cuidadores de personas con enfermedad crónica de la comunidad de Virgen del Carmen La Era, tal como se han demostrado en los resultados anteriormente discutidos.

\section{CONCLUSIONES}

- La Implementación de una Red de Apoyo Social Adventista tiene un efecto significativo en el nivel de conocimiento de los familiares cuidadores de 
personas con enfermedad crónica del Centro Poblado Virgen del Carmen - La Era.

- La Implementación de una Red de Apoyo Social Adventista tiene un efecto significativo en el nivel de valor de los familiares cuidadores de personas con enfermedad crónica del Centro Poblado Virgen del Carmen - La Era.

- La Implementación de una Red de Apoyo Social Adventista tiene un efecto significativo en el nivel de paciencia de los familiares cuidadores de personas con enfermedad crónica del Centro Poblado Virgen del Carmen - La Era.

- La Implementación de una Red de Apoyo Social Adventista tiene un efecto significativo en el nivel de habilidad de cuidado de los familiares cuidadores de personas con enfermedad crónica del Centro Poblado Virgen del Carmen - La Era.

\section{REFERENCIAS BIBLIOGRÁFICAS}

1. Alvarado, A. (2007).Adquiriendo habilidad en el cuidado "de la incertidumbre al nuevo compromiso" (Versión electrónica). Aquichian, 7 (01), 25-36. Universidad de La Sabana Colombia. Disponible en http://redalyc.uaemex.mx/src/inicio/ArtPdfRed. jsp?iCve $=74170103$.

2. Anderson, R., y Cols. (2000). Coregivers Unmeet needs for support in caring for functionally impaired elderly person. Journal of Health for the poor and underserved, 11.(4), 412.

3. Anyud, K. (2005). Confiabilidad del instrumento traducido al español: Inventario de habilidad del cuidado. Universidad Nacional de Colombia. 2005; 770 .

4. Asociación General de la Iglesia Adventista del Séptimo Día (2005). Manual de la Iglesia. Buenos Aires. Asociación Casa Editora Sudamericana.

5. Asociación Peruana del Sur. Sobre los Adventistas (s.f).Extraidoel20Abril2010de http://www.apcsur. org/contenidos.php?menu $=$ adventistas $\&$ cid $=8$

6. Barrera O. L, Galvis L. CR, Moreno F. ME, Pinto A. N, Pinzón R. ML, Romero G. E, Sánchez H. B. (2006). La habilidad de cuidado de los cuidadores familiares de personas con enfermedad crónica. Invest. educ. enferm.,24(1), 35-46.

7. Barrera, L. (2005). Investigación habilidad de cuidado de los cuidadores de personas en situación de enfermedad crónica. En Grupo de cuidado. La Investigación y el cuidado en América Latina. Facultad de Enfermería Universidad Nacional de Colombia. UNIBIBLOS, 2005, 271-280.
8. Clemente, M., Tartaglini, M., Stéfani., D. (2008). Apoyo social. Bienestar psicológico y salud percibidas en personas de edad. Perspectivas en Psicología. Rev. Psicología y Ciencias Afines. Universidad Nacional de Mar del Plata, 5 (2), 47-53.

9. Corera, C. (2002). Los servicios sociales de atención primaria ante la desigualdad y los nuevos procesos de exclusión, en Memoria del Congreso de Servicios Sociales de Atención Primaria. 20 años de Servicios Sociales en el Ayuntamiento de Murcia. Murcia.

10. Díaz, JC., Rojas, M. (2009). Cuidando al cuidador: efectos de un programa educativo (Versión electrónica). Aquichan, 9 (1), 73-92. Universidad de La Sabana Colombia. Disponible en http:// redalyc.uaemex.mx/src/inicio/ArtPdfRed. jsp?iCve $=74111465008$

11. División Sudamericana de la Asociación General de los Adventistas del Séptimo Día (2008). Reglamentos Eclesiástico Administrativos. Buenos Aires. Asociación Casa Editora Sudamericana.

12. Dominguez, C. (2003). La percepción del cuidar. Revista Rol de Enfermería. Bogotá. Nº127, 25 27.

13. García, L. y Pardo, C. (1996). Factores asociados a la ansiedad y toma de psicofármacos en cuidadores de pacientes discapacitados. Atención Primaria, 7 , 395-98.

14. García, M., Mateo I. y Eguiguren, A. (2004). El sistema informal de cuidados en clave de desigualdad. Gaceta Sanitaria, 18, 132-139. 
15. García, M., Mateo, I. y Gutiérrez, P. (1999). Cuidados y cuidadores en el sistema informal de salud. Granada: Escuela Andaluza de Salud Pública e Instituto Andaluz de de la Mujer.

16. Gaut D. (1993). Comprendiendo el significado de la enfermedad crónica: Un pre - requisito para cuidar. A Global Agenda for Caring. National League for nursig press. New York., 281-291.

17. Geeneral Conference Corpòration of Seventh Day Adventist (2009). Seventh Day Adventist Yearbook. U.S.A. Review and Herald Publishing Association Hagertown.

18. Gil, E., Escudero, M., Prieto, M. y Frias, A. (2005). Vivencia, expectativas y demandas de cuidadoras informales de pacientes en procesos de enfermedad de larga duración. Enfermería Clínica, 15(4), 220-26.

19. Gonzales, D. (2006). Habilidad de cuidado de los cuidadores familiares de personas en situación de enfermedad crónica por Diabetes Mellitus (Versión electrónica). Avances en enfermería, 24(2):2837. Disponible en http://bases.bireme.br/cgi-bin/ wxislind.exe/iah/online/?IsisScript=iah/iah.xis\&src $=$ google $\&$ base $=$ LILACS\&lang $=$ p\&nextAction $=\ln$ $\mathrm{k} \&$ exprSearch=480362\&indexSearch=ID

20. Hunt, CK. (2003). Concepts in caregiver research. J. Nurs Scholarsh, 35(1), 27- 32.

21. Kaplan, J. R., Manuck, S., Clarkson, T., Lusso, F., Taub, D. y Miller, E.W. (1983). Social stress and atherosclerosis in normocholesterolemic monkeys. Science, 220, 733-735.

22. Lee, S., Colditz, G., Berkman, L. y Kawachi, I. (2003). Caregiving and risk coronary heart disease in U.S. women: A prospective study. American Journal of Preventive Medicine, 24(2), 113-119.

23. Leininger, M. (1981). Transcultural nursing: Its progress and its future. Nursing and Health Care, 2(7), 365-371.

24. López E.(1998). Enfermería en Cuidados Paliativos. Editorial Médica Panamericana, S.A. Madrid, España.

25. Martin, E. y Davila, L.(2008). Redes de apoyo social y adaptación de los menores en acogimiento residencial. Psicotema.20 (2), 229-235.
26. Mateo, I., Millán, A., García, M., Gonzalo, E. y López, L. (2000). Cuidadores familiares de personas con enfermedad neurodegenerativa: perfil, aportaciones e impacto de cuidar. Atención Primaria, 26(3), 25-34.

27. Méndez, E. y Cruz, L. (2008). Redes sociales de apoyo y persona adulta mayor: Costa Rica. Documento preparado para el I Informe del Estado de Situación de la Persona Adulta Mayor en Costa Rica, UCR/ CONAPAM.

28. Ministerio de Salud (2009, Noviembre 15). (MINSA). Obesidad, sobre peso e hipertensión incrementan enfermedades del corazón y cáncer. Nota de prensa. Extraido de: http://www.minsa. gob.pe/ocom/prensa/notadeprensa.asp?np codigo $=8160 \&$ mes $=11 \&$ anio $=2009$.

29. Montalvo, A., Flórez, I. y Stavro de Vega, D. (2008). Cuidando a cuidadores familiares de niños en situación de discapacidad (Versión electrónica). Aquichan, 8(2), 197-21. Universidad de La Sabana Colombia. Disponible en http://redalyc.uaemex. $\mathrm{mx} / \mathrm{src} /$ inicio/ArtPdfRed.jsp?iCve=74180207.

30. Moral, M., Juan, J. y López, M. (2003). Perfil y riesgo de morbilidad psíquica en cuidadores de pacientes ingresados en su domicilio. Atención Primaria, 32(2), 77-83.

31. Moreno M., Náder, A, López de MC. (2004). Relación entre las características de los cuidadores familiares de pacientes con discapacidad y la percepción de su estado de salud. Avances en enfermería, (22):27-38.

32. Nkongho, N. (1999). The Coring ability inventory instrickland. Mecesurement of Nursing out comes. V 4.

33. Novel, G., Lluch, M., Rourera, A. (1995). Aspectos psicosociales del paciente crónico, en G. Novel et al, Enfermería Psicosocial y Salud Mental (pp 239- 244). Barcelona: Masson.

34. Organización Mundial de la Salud (2008). $61^{\circ}$ Asamblea Mundial de la Salud. Informe de secretaría. Prevención y control de las enfermedades no transmisibles: aplicación de la estrategia mundial. Washington, DC: Secretaría.

35. Organización Mundial de la Salud (2010). Enfermedades crónicas. Temas de Salud. Extraído el 15 Marzo, 2010, de http://www.who.int/topics/ chronic_diseases/es. 
36. Organización Mundial de la Salud (2010). Prevención de las enfermedades crónicas. Enfermedades Crónicas y Promoción de la Salud. Extraido el 15 Marzo, 2010, de: http://www.who. int/chp/chronic_disease_report/part1/es/index. html

37. Organización Mundial de la salud. (1980). International Classification of Impairments, Disability and Handicaps (ICIDH). Ginebra: OMS.

38. Paleo, N., Falcón, N. y Rodríguez, L. (2005). Sobrecarga de los cuidadores de pacientes con demencia de Alzheimer. Interpsiquis. Extraido Octubre 24, 2008, de http://weblog.maimonides. edu/gerontologia2004/archives/000919.html.

39. Philip, I., Young, J. (1988). Audit of support given to lay carers of the demented elderly by a primary team. J Roy Coll Gen Pract, 38, 153-58.

40. Pinto, N., Sanchez, B. (2001). El reto de los cuidadores: familiares de perdonas en situación de enfermedad. Cuidado y práctica de enfermería. Grupo de Cuidado. Facultad de Enfermería. Universidad Nacional de Colombia.

41. Pinto, N., Sanchez, B. (2001). El reto de los cuidadores: familiares de perdonas en situación de enfermedad". En cuidado y práctica de enfermería. Grupo de Cuidado. Facultad de Enfermería. Universidad Nacional de Colombia.

42. Puga, D. Rosero-Bixby, L. Glaser, K. y Castro, T. (2007). Red social y salud del adulto mayor en perspectiva comparada: Costa Rica, España e Inglaterra. Población y Salud en Mesoamérica. 5
(1), artículo1. Centro Centroamericano de Población de Población. Universidad de Costa Rica.

43. Sánchez B. (2000). La experiencia de ser cuidadora de una persona en situación de enfermedad crónica. Investigación y educación en enfermería, 19(2):36-51.

44. Sánchez, C. (2000). Gerontología Social. Editorial Espacio. Buenos Aires, Argentina.

45. Schultz, R. y Beach, SR. (1999). Caregiving as a risk factor for mortality. The caregiver health effects study. JAMA, 282, 2215-2229.

46. Vega, Mendoza, Ureña y Villamil (2008). Efecto de un programa educativo en la habilidad de cuidado de los cuidadores familiares de personas en situación crónica de enfermedad, Rev. Ciencia y Cuidado. Extraido el 19 Diciembre 2010 de http://dialnet. unirioja.es/servlet/articulo?codigo $=2884811$

47. Velásquez, A (2009). La carga de enfermedad y lesiones en el Perú y las prioridades del plan esencial de aseguramiento universal. Rev. Perú Med. Exp. Salud Pública. Extraido el 15 Abril 2010 de http://www.scielo.org.pe/pdf/rins/v26n2/ a15v26n2.pdf

48. Watson, J. (1979). Nursing: The philosophy and science of caring. Boston: Little Brown.

49. WHO. (2002). Lessons for Long-Term Care Policy. The Cross-Clluster Iniciative on Long_Term Care. Geneve: World Health Organization.

50. Wilson, S, Y Cols. (1999). Desarrollando confianza reciproca en la relación entre cuidador y receptor del cuidado". Qualitative Health Research (Quail Health Res) (p.p 446). 